\title{
Observation of Intensity Statistics of Light Transmitted Through 3D Random Media
}

\author{
Tom Strudley, ${ }^{1, *}$ Duygu Akbulut, ${ }^{2,3, *}$ Willem L. Vos, ${ }^{2}$ \\ Ad Lagendijk, ${ }^{2}$ Allard P. Mosk, ${ }^{2}$ and Otto L. Muskens ${ }^{1, \dagger}$ \\ ${ }^{1}$ Faculty of Physical and Applied Sciences, University of Southampton, Highfield, Southampton SO17 1BJ, UK \\ ${ }^{2}$ Complex Photonic Systems (COPS), MESA+ Institute for Nanotechnology, \\ University of Twente, P.O.Box 217, 7500 AE Enschede, The Netherlands \\ ${ }^{3}$ Present address: ASML, Flight Forum 1900, 5657 EZ Eindhoven, The Netherlands
}

compiled: September 30, 2014

\begin{abstract}
We experimentally observe the spatial intensity statistics of light transmitted through three-dimensional isotropic scattering media. The intensity distributions measured through layers consisting of zinc oxide nanoparticles differ significantly from the usual Rayleigh statistics associated with speckle, and instead are in agreement with the predictions of mesoscopic transport theory, taking into account the known material parameters of the samples. Consistent with the measured spatial intensity fluctuations, the total transmission fluctuates. The magnitude of the fluctuations in the total transmission is smaller than expected on the basis of quasi-onedimensional (1D) transport theory, which indicates that quasi-1D theories cannot fully describe these open three-dimensional media.
\end{abstract}

OCIS codes: $\quad$ (030.6600) Statistical Optics; (110.7050) Turbid Media; (290.4210) Multiple Scattering

http://dx.doi.org/10.1364/XX.99.099999

Understanding the flow of light in three-dimensional (3D) scattering environments is important for a variety of applications ranging from new developments in biomedical imaging [1] to energy harvesting [2], spectroscopy [3, 4], information control [5] and lighting [6]. Often, light transport in such media can be approximated as a series of uncorrelated, random events. However, interference between scattered fields can give rise to mesoscopic effects that can reveal valuable information on the properties of the medium and the transport process. In quantum transport theory for electrons, mesoscopic correlations originate from the crossing of many possible trajectories inside the medium [7]. The analogy between electrons and matter waves with classical waves such as light and sound allows a direct mapping of concepts from mesoscopic transport theory. The crossing probabilities of wave paths and the resulting correlations are described by a single parameter, the dimensionless conductance $g$, which is equal to the average number of open transmission eigenchannels. In a waveguide geometry, $g$ is defined as $g=N\left\langle T_{a}\right\rangle$, with $N$ the total number of transmission eigenchannels that light in the incident free-space modes can couple to and $\left\langle T_{a}\right\rangle \approx l_{\mathrm{tr}} / L$ the en-

\footnotetext{
* These authors contributed equally

† Corresponding author: O.Muskens@soton.ac.uk
}

semble averaged transmission probability of light, with $l_{\text {tr }}$ the transport mean free path and $L$ the thickness.

Statistical methods have been widely employed in the study of wave transport through disordered systems to extract mesoscopic transport contributions [8-22]. The dimensionality of the experiment is of paramount importance. Quasi one-dimensional (1D) waveguides allow the direct observation of light propagation in the strongly mesoscopic regime, including Anderson localization $[11,12]$. Deviations from Rayleigh statistics were observed in the crossover from the $1 \mathrm{D}$ to the quasi$1 \mathrm{D}$ regime using stacks of glass slides [23] and in $2 \mathrm{D}$ photonic crystal waveguides containing localized modes $[18,19]$. The case of 3D random media is of special interest, as only in 3D a phase transition to localization is expected. In 3D materials however, the effects of interference are generally much weaker because the large available phase space leads to a reduced probability for trajectories to cross. Mesoscopic effects for light in 3D media are therefore generally quite subtle and difficult to measure $[9,21,22,24]$. Intensity statistics deviating from Rayleigh statistics in 3D have only been observed in strongly anisotropic disordered mats of semiconductor nanowires [17]. The observation of transmission statistics beyond the Rayleigh regime is a crucial test for the extension of mesoscopic transport theory to these inherently open 3D media.

Here we present measurements of the intensity fluctu- 


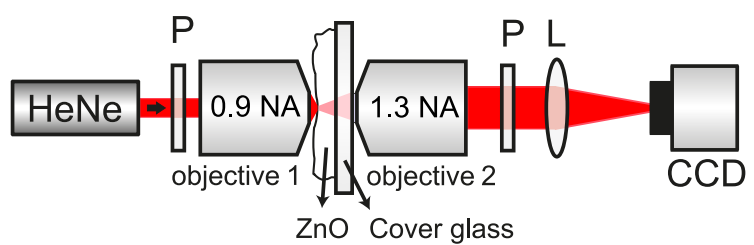

Fig. 1. Experimental setup. HeNe: laser $(=\lambda=632.8 \mathrm{~nm}$, $\mathrm{P}=5 \mathrm{~mW})$. ZnO: sample. Objective 1: $100 \times 0.9$-NA objective. Objective 2: $100 \times 1.3-\mathrm{NA}$ oil immersion objective. L: $200 \mathrm{~mm}$ focal length lens. P: polarizer. CCD: camera sensor.

ations in the transmission of light through 3D layers of $\mathrm{ZnO}$ particles with an average size of $200 \mathrm{~nm}$. Transmission was recorded spatially using a high numerical aperture (NA) transmission microscope as shown in Fig. 1 and described in more detail in Ref. [17]. Light from a Helium-Neon laser was focused on the incident surface of the sample. The spatial distribution of transmitted light at $\mathrm{ZnO}$-glass interface was imaged onto a camera, in a cross-polarized configuration.

We performed measurements in two configurations, 'in-focus' (IF) and 'out-of-focus' (OF) illumination, corresponding to illumination spots with a width at $1 / e$ of less than $0.5 \mu \mathrm{m}$ and $25 \mu \mathrm{m}$, respectively. In each configuration, 1000 images were captured per dataset, translating the sample over $1 \mu \mathrm{m}$ for each consecutive image. At this distance, the speckle patterns of any two consecutive images were found to be completely different. A total number of 6 datasets per sample were recorded in a procedure identical to that followed in Ref. [17], in brief: For each dataset, the captured images were averaged to obtain an average spatial intensity distribution. The total transmitted intensity for each sample position was obtained by summing the total intensity in the corresponding image. In order to divide out any sample variations over long length scales, the total transmission was normalized to a moving average over $10 \mu \mathrm{m}$. A constant background corresponding to the dark counts of the camera was subtracted from all captured images. Finally, each image was cropped to the area of interest and divided pixel by pixel by the average intensity image to obtain the normalized intensity $s_{a b}=t_{a b} /\left\langle t_{a b}\right\rangle$, where $a$ denotes an input mode and $b$ an output mode, and the brackets denote ensemble averaging. This procedure divides out the envelope in the intensity due to diffusion. All intensities for the 6 datasets were collected into a single histogram, to obtain $P\left(s_{a b}\right)$ vs $s_{a b}$. After collection of the IF histogram, the illumination objective was moved out of focus by $25 \mu \mathrm{m}$ and the measurement was repeated in this 'out-of-focus' (OF) configuration. The number of transmission channels addressed by the incident field is large when OF and small when IF. Thus, the measurement made when OF serves as a reference for the case of large $g$ and negligible mesoscopic corrections, whereas the measurements performed when IF are expected to give rise to strong mesoscopic fluctuations.

In Fig. 2a-c we show the spatial intensity histograms
$P\left(s_{a b}\right)$ vs $s_{a b}$ using the data captured IF and OF for three samples A-C. The OF data is expected to follow Rayleigh statistics, $P_{R}\left(s_{a b}\right)=\exp \left(-s_{a b}\right)$, however due to experimental aberrations the tail of the OF distribution is slightly suppressed. For reduced speckle visibility, the exact intensity histogram is described by the gamma-distribution [25], which in the current regime is well approximated by a single exponential with increased slope according to $P_{R}^{\prime}\left(s_{a b}\right)=c^{-1} \exp \left(-s_{a b} / c\right)$ (dashed lines, green in Fig. 2a-c), with $c \simeq 0.95$ the experimental speckle contrast parameter. In comparison to OF, the histograms of the data captured IF show a heavy tail at high speckle intensities, which is not present in the data captured OF and therefore is a direct signal of mesoscopic fluctuations. We compared the histogram of $s_{a b}$ in Fig. 2 for IF with a theoretical model for the mesoscopic distribution [26-28], which can be modified to include finite contrast by taking into account the reduced single channel transmission, resulting in

$$
P\left(s_{a b}\right)=P_{R}^{\prime}\left(s_{a b}\right)\left\{1+\frac{1}{3 g}\left(\frac{s_{a b}^{2}}{c^{2}}-4 \frac{s_{a b}}{c}+2\right)\right\} .
$$

A robust method to analyze distributions is to fit their moments. An analytical expression of the moments of the intensity distribution was developed by Kogan et al. [28]. Following the above reasoning, we introduce the finite contrast in this expression by approximating $\left\langle t_{a b}\right\rangle=c\left\langle s_{a b}\right\rangle$ and normalizing the first moment $\left\langle s_{a b}\right\rangle$ which yields

$$
<s_{a b}^{N}>=c^{-1} N !\left(\frac{c}{2 i g^{1 / 2}}\right)^{N} H_{N}\left(i g^{1 / 2}\right),
$$

with $H_{N}$ the Hermite polynomial of order $N$. The moments of the measured distributions are shown in Fig. 2d-f. We see a large difference between the OF (black diamonds) and IF (red dots) moments. The analytical expression (2) was fitted to the first five moments of the data, using the contrast $c$ and the dimensionless conductance $g$ as the free parameters. The fits to the first 5 moments of the OF histogram resulted in $g$ values on the order of $10^{5}$, indicating that we can not distinguish them from a Rayleigh distribution (infinite $g$ ). We use the $\mathrm{OF}$ fits to obtain the contrast values $c$ with values indicated in the figure. For moments $N>6$, the statistical variations due to rare events give rise to a large uncertainty and we did not include these moments in the fits. The first 5 moments of the IF distribution were fitted using only $g$ as a free parameter, with the experimental contrast parameter $c$ fixed by the OF data. Best fits (shown in Fig. 2d-f) were found for values of $g$ in the range $35-65$. The fits agree with the data up to the $10^{\text {th }}$ moment, indicating that mescosopic theory describes the observed intensity fluctuations well. We did not observe evidence of other, nonuniversal contributions such as for example $C_{0}$ correlations [29].

A 3D medium differs from a waveguide in the fact that energy can spread out in the transverse direction. 

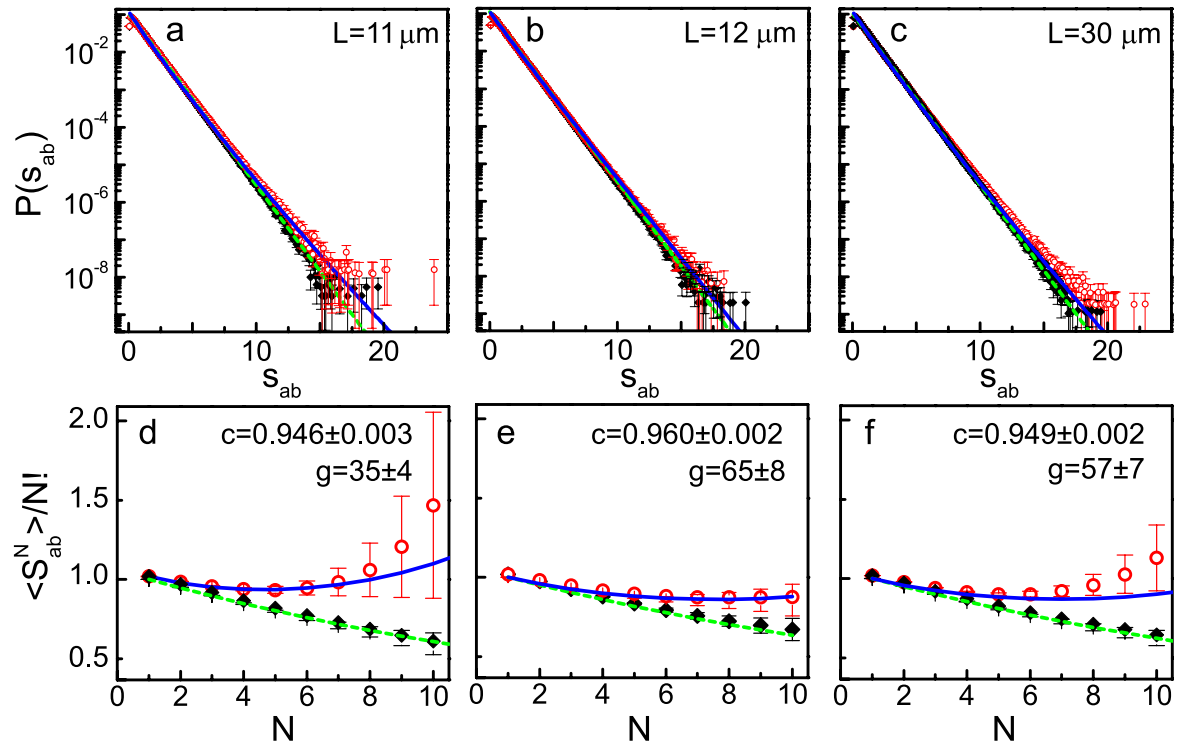

Fig. 2. [wide figure] (a-c) Histograms of the intensity distribution $P\left(s_{a b}\right)$ of fields transmitted through samples labeled A-C. Data points: Mean of normalized histograms of six different datasets captured IF (open dots, red) and OF (diamonds, black). Error bars: standard error of the normalized histograms based on pixel counts. Dashed line, green: Rayleigh statistics with reduced contrast $c$ obtained from moment fits. Solid line, blue: Plot of Eq. 1 with $g$ from fits of (d-f). (d-f) Moments of the intensity distributions of transmitted fields for IF (open dots, red) and OF (diamonds, black) configurations. Dashed line, green: Fits of first 5 OF moments using Rayleigh theory with reduced contrast $c$ as indicated in figures. Solid line, blue: Fits of IF moments with Eq. 2 for $g=35 \pm 4$ (d), $g=65 \pm 8$ (e) and $g=57 \pm 7$ (f).

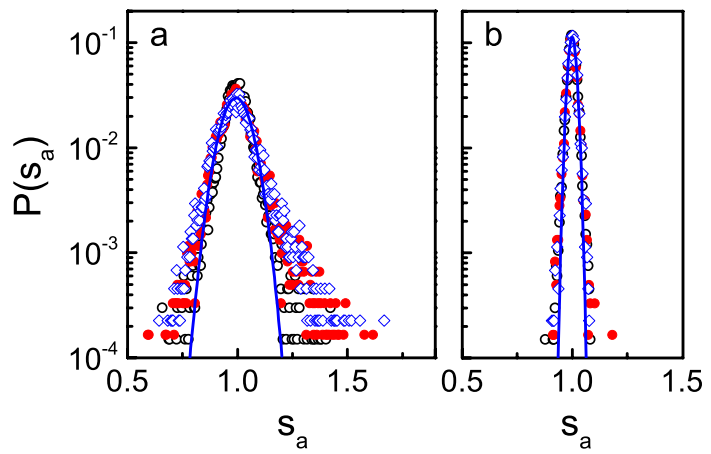

Fig. 3. Histograms of the total transmission $s_{a}$ for (a) IF and (b) OF, for $\mathrm{ZnO}$ samples $\mathrm{A}$ (dots, red), B (open dots, black), and C (open diamonds, blue). Lines: Gaussian fits. Variances of distributions are shown in Table 1.

This geometry has been modeled as a waveguide of expanding width $[21,22]$ with an effective conductance parameter $g$ that increases with $N$ and $l_{\text {tr }}$, but saturates with increasing sample thickness $L$. In the case of an incident beam that is tightly focussed on the sample the expanding waveguide model predicts a conductance $g=\left(8 l_{t r} k^{2} / 15\right) w_{\min }$. Here $k$ represents the wavevector inside the medium and $w_{\min }=a l_{\mathrm{tr}}$ is the minimum width of the incident spot inside the sample, where $a$ is a constant expected to be close to unity. A later elegant approach [30] obtains similar results. We use the previously measured values $l_{\mathrm{tr}}=0.7 \pm 0.2 \mu \mathrm{m}$ and $n_{\mathrm{eff}}=1.4 \pm 0.1$ [31] in the expanding waveguide formal- ism and find $g$ in the range $(50.47 \pm 29.73) a$, in good agreement with the fits to the histograms.

In addition to the spatial intensity statistics, our experimental configuration provides measurements of the total transmitted intensity. For this purpose, we integrated the total counts in each camera image, which we normalized to the ensemble average to obtain the normalized total transmission $s_{a}$. Figure 3 shows the IF and OF distributions $P\left(s_{a}\right)$ for the two samples under study. The total transmission is the sum of a large number of independent speckle spots, and the exponential distribution converts to a Gaussian with a variance inversely proportional to the number of independent transmission channels $g$. The OF data shows a correspondingly narrow distribution, which is very similar $\left(\operatorname{var}\left(s_{a}^{\mathrm{OF}}\right) \simeq 4 \times 10^{-4}\right)$ for the three samples under study. The IF data showed a much broader distribution indicating a reduction of the number of contributing 'open' channels $g$, again with little variation between the three samples. The measured variances for both the $s_{a}$ and the $s_{a b}$ distributions are summarized in Table 1 . For the latter we corrected the IF variance for the finite speckle contrast by normalizing to the OF variance (speckle contrast does not affect the fluctuations of $s_{a}$. Error bars were obtained by calculating the individual variances for the 6 independent datasets taken at different positions on each sample. The significant differences of the variance values between samples $\mathrm{A}$ and $\mathrm{B}$, of almost equal thickness, are most likely caused by intrinsic variations in density and morphology between similarly prepared 


\begin{tabular}{c|cccccc} 
Sample & $\mathrm{L}(\mu \mathrm{m})$ & $g_{\mathrm{fit}}$ & $\left(\frac{\operatorname{var}\left(s_{a b}^{\mathrm{IF}}\right)}{\operatorname{var}\left(s_{a b}^{\mathrm{OF}}\right)}-1\right)$ & $\operatorname{var}\left(s_{a}^{\mathrm{IF}}\right)$ & ratio & p-value \\
\hline $\mathrm{A}$ & 11 & $35 \pm 4$ & $(3.1 \pm 0.3) \times 10^{-2}$ & $(7.1 \pm 0.6) \times 10^{-3}$ & $4.4 \pm 0.6$ & 0.0037 \\
$\mathrm{~B}$ & 12 & $65 \pm 8$ & $(2.1 \pm 0.5) \times 10^{-2}$ & $(4.2 \pm 0.5) \times 10^{-3}$ & $5.0 \pm 1.4$ & 0.038 \\
$\mathrm{C}$ & 30 & $57 \pm 7$ & $(1.6 \pm 0.5) \times 10^{-2}$ & $(9.9 \pm 1.5) \times 10^{-3}$ & $1.6 \pm 0.6$ & 0.26 \\
\hline
\end{tabular}

Table 1. Fitted values of $g$ obtained from moments of spatial intensity distribution, and measured variances of the intensity distributions for samples A-C. Error bars denote standard error of mean taken over 6 data sets for each sample. P-value indicates the statistical significance of the deviation of the $\operatorname{var}\left(s_{a b}\right) / \operatorname{var}\left(s_{a}\right)$ ratio from 2, obtained using a Student's t-test.

samples, not to extrinsic properties such as thickness. For the quasi-1D geometry of a waveguide, random matrix theory predicts the relation $\operatorname{var} s_{a b}=1+2 \operatorname{var}\left(s_{a}\right)$ [11]. It is unknown whether this theoretical relation can be extended in unmodified form for vector waves in 3D media. The results in Table 1 indicate that the variance ratio fluctuates and for individual samples may be significantly different from the quasi-1D value of 2 .

In conclusion, we have presented measurements of the intensity statistics of light transmitted through threedimensional isotropic $\mathrm{ZnO}$ scattering media. The results show deviations from Rayleigh statistics. Using an analysis of the moments of the distribution we obtain values of the dimensionless conductance $g$ of around 40, in agreement with predictions based on previously reported sample parameters. This is the first direct observation through intensity statistics of strong mesoscopic effects of light transmitted through isotropically scattering 3D samples. Our results show a good agreement of the modes of the intensity distribution with transport theory. However, the ratio between the variances of the total transmission and the speckle transmission differs from the predictions of quasi-1D theory. Compared to random waveguides where much is known about transport in the few channel regime, more studies are needed to elucidate wave transport in 3D materials. Ultimately, mesoscopic effects affect important applications such as wavefront shaping and time reversal methods [5], and spectroscopy $[3,4]$. Our results show that the regime where mesoscopic fluctuations are measurable is accessible using conventional scattering materials, opening up new avenues for experimental investigation.

This work is supported by FOM-NWO, FOM program "Stirring of light" and ERC grant 279248. O.M. acknowledges support by EPSRC through fellowship $\mathrm{EP} / \mathrm{J} 016918 / 1$.

\section{References}

[1] J. Jang et al., Opt. Express 21, 2890 (2013).

[2] K. Vynck, M. Burresi, F. Riboli, and D. S. Wiersma, Nature Mater. 11, 1017 (2012).

[3] B. Redding, S. F. Liew, R. Sarma, and H. Cao, Nat. Photon. 7, 746 (2013)

[4] M. Mazilu, T. Vettenburg, A. Di Falco, and K. Dholakia, Opt. Lett. 39, 96 (2014)

[5] A.P. Mosk, G. Lerosey, A. Lagendijk, and M. Fink, Nature Photon. 6, 283 (2012).

[6] V. Y. F. Leung et al., Opt. Express 22, 8190 (2014).

[7] E. Akkermans and G. Montambaux, Mesoscopic physics of electrons and photons, Cambridge University Press, 2007.

[8] N. Garcia and A. Z. Genack, Phys. Rev. Lett. 63, 1678 (1989).

[9] J. F. de Boer, M. C. W. van Rossum, M. P. van Albada, Th. M. Nieuwenhuizen, A. Lagendijk, Phys. Rev. Lett. 73, 2567 (1994).

[10] M. Stoytchev and A. Z. Genack, Phys. Rev. Lett. 79, 309 (1997).

[11] A. A. Chabanov, M. Stoytchev, and A. Z. Genack, Nature 404, 850 (2000).

[12] A. Peña, A. Girschik, F. Libisch, S. Rotter, A. A. Chabanov, Nat. Commun. 5, 3488 (2014)

[13] S. Balog, P. Zakharov, F. Scheffold, and S. E. Skipetrov, Phys. Rev. Lett. 97(10),103901 (2006)

[14] H. Hu, A. Strybulevych, J. H. Page, S. E. Skipetrov, and B. A. van Tiggelen, Nature Phys. 4, 945 (2008).

[15] S. Smolka, H. Thyrrestrup, L. Sapienza, T. B. Lehmann, K. R. Rix, L. S. Froufe-Perez, P. D. Garcia, P. Lodahl, New J. of Phys. 13, 063044 (2011).

[16] R. Uppu and S. Mujumdar, Phys. Rev. A 87, 013822 (2013).

[17] T. Strudley, T. Zehender, C. Blejean, E. P. A. M. Bakkers, O. L. Muskens, Nat. Photon. 7, 413 (2013).

[18] F. Riboli, N. Caselli, S. Vignolini, F. Intonti, K. Vynck, P. Barthelemy, A. Gerardino, L. Balet, L. H. Li, A. Fiore, M. Gurioli, D. S. Wiersma, Nat. Mater. 13, 720 (2014).

[19] P. D. García, S. Stobbe, I. Söllner, and P. Lodahl, Phys. Rev. Lett. 109, 253902 (2012).

[20] Y. Bromberg and H. Cao, Phys. Rev. Lett. 112, 213904 (2014).

[21] F. Scheffold, W. Härtl, G. Maret, and E. Matijević, Phys. Rev. B 56, 10942 (1997).

[22] F. Scheffold, and G. Maret, Phys. Rev. Lett. 81, 5800 (1998).

[23] J. Park, S. Zhang, and A. Z. Genack, Phys. Rev. E 82, 045101 (2010).

[24] J. F. de Boer, M. P. van Albada, and A. Lagendijk, Phys. Rev. B 45, 658 (1992).

[25] S. E. Skipetrov, J. Peuser, R. Cerbino, P. Zakharov, B. Weber, F. Scheffold, Opt. Expr. 18, 14519 (2010).

[26] N. Shnerb and M. Kaveh, Phys. Rev. B 43, 1279 (1991).

[27] Th. M. Nieuwenhuizen and M. C. W. van Rossum, Phys. Rev. Lett. 74, 2674 (1995).

[28] E. Kogan, M. Kaveh, R. Baumgartner, and R. Berkovits, Phys. Rev. B, 48, 9404 (1993).

[29] W. K. Hildebrand, A. Strybulevych, S. E. Skipetrov, B. A. van Tiggelen, J. H. Page, Phys. Rev. Lett. 112, 073902 (2014).

[30] A. García-Martín, F. Scheffold, M. Nieto-Vesperinas, and J. J. Sáenz, Phys. Rev. Lett. 88, 143901 (2002). 
[31] I. M. Vellekoop, E. G. van Putten, A. Lagendijk, and A.

P. Mosk, Opt. Express 16, 67 (2008). 
[1] J. Jang, J. Lim, H. Yu, H. Choi, J. Ha, J.-H. Park, W.-Y. Oh, W. Jang, S. Lee, Y. Park, Complex wavefront shaping for optimal depth-selective focusing in optical coherence tomography, Optics Express 21, 2890 (2013).

[2] K. Vynck, M. Burresi, F. Riboli, D. S. Wiersma, Photon Management in Two-Dimensional Disordered Media, Nat. Mater. 11, 1017-1022 (2012).

[3] B. Redding, S. F. Liew, R. Sarma, H. Cao, Compact spectrometer based on a disordered photonic chip, Nat. Photon. 7, 746 (2013).

[4] M. Mazilu, T. Vettenburg, A. Di Falco, K. Dholakia, Random super-prism wavelength meter, Opt. Lett. 39, 96 (2014).

[5] A.P. Mosk, G. Lerosey, A. Lagendijk, M. Fink, Controlling waves in space and time for imaging and focusing in complex media, Nature Photon. 6, 283 (2012).

[6] V. Y. F. Leung, A. Lagendijk, T. W. Tukker, A. P. Mosk, W. L. IJzerman, W. L. Vos, Interplay between multiple scattering, emission, and absorption of light in the phosphor of a white lightemitting diode, Opt. Express 22, 8190 (2014).

[7] E. Akkermans and G. Montambaux. Mesoscopic physics of electrons and photons. Cambridge University Press, 2007.

[8] N. Garcia and A. Z. Genack. Crossover to strong intensity correlation for microwave radiation in random media. Phys. Rev. Lett. 63, 1678-1681 (1989).

[9] J. F. de Boer, M. C. W. van Rossum, M. P. van Albada, Th. M. Nieuwenhuizen, A. Lagendijk Probability distribution of multiple scattered light measured in total transmission. Phys. Rev. Lett. 73(19),2567-2570 (1994).

[10] M. Stoytchev and A. Z. Genack. Measurement of the probability distribution of total transmission in random waveguides. Phys. Rev. Lett. 79, 309-312 (1997).

[11] A. A. Chabanov, M. Stoytchev, A. Z. Genack. Statistical signatures of photon localization. Nature 404, 850-853 (2000).

[12] A. Peña, A. Girschik, F. Libisch, S. Rotter, A. A. Chabanov, The single-channel regime of transport through random media, Nat. Commun. 5, 3488 (2014).

[13] S. Balog, P. Zakharov, F. Scheffold, S. E. Skipetrov, Photocount statistics in mesoscopic optics, Phys. Rev. Lett. 97(10),103901 (2006).

[14] H. Hu, A. Strybulevych, J. H. Page, S. E. Skipetrov, B. A. van Tiggelen, Localization of ultrasound in a three-dimensional elastic network, Nat. Phys. 4, 945-948 (2008).

[15] S. Smolka, H. Thyrrestrup, L. Sapienza, T. B. Lehmann, K. R. Rix, L. S. Froufe-Perez, P. D. Garcia, P. Lodahl, Probing statistical properties of Anderson localization with quantum emitters, New Journal of Physics 13 (6), 063044 (2011).

[16] R. Uppu, S. Mujumdar, Dependence of the Gaussian-Lévy transition on the disorder strength in random lasers, Phys. Rev. A 87, 013822 (2013).
[17] T. Strudley, T. Zehender, C. Blejean, E. P. A. M. Bakkers, O. L. Muskens, Mesoscopic light transport by very strong collective multiple scattering in nanowire mats. Nat. Photon., 7, 413-418 (2013).

[18] F. Riboli, N. Caselli, S. Vignolini, F. Intonti, K. Vynck, P. Barthelemy, A. Gerardino, L. Balet, L. H. Li, A. Fiore, M. Gurioli, D. S. Wiersma, Engineering of light confinement in strongly scattering disordered media, Nat. Mater. 13, 720-725 (2014)

[19] P. D. García, S. Stobbe, I. Söllner, P. Lodahl, Nonuniversal Intensity Correlations in a Two-Dimensional Anderson-Localizing Random Medium, Phys. Rev. Lett. 109, 253902 (2012).

[20] Y. Bromberg, H. Cao, Generating Non-Rayleigh Speckles with Tailored Intensity Statistics, Phys. Rev. Lett. 112, 213904 (2014).

[21] F. Scheffold, W Härtl, G. Maret, and E. Matijević. Observation of long-range correlations in temporal intensity fluctuations of light. Phys. Rev. B, 56,10942-10952 (1997).

[22] F. Scheffold and G. Maret, Universal Conductance Fluctuations of Light, Phys. Rev. Lett. 82(26), 5800-5803 (1998).

[23] J. Park, S. Zhang, A. Z. Genack. Intensity statistics and photon localization beyond one dimension. Phys. Rev. E, 82,045101 (2010).

[24] J. F. de Boer, M. P. van Albada, A. Lagendijk. Transmission and intensity correlations in wavepropagation through random-media. Phys. Rev. B, 45,658-666 (1992).

[25] S. E. Skipetrov, J. Peuser, R. Cerbino, P. Zakharov, B. Weber, F. Scheffold, Noise in laser speckle correlation and imaging techniques, Optics Express, 18(14), 14519-14534 (2010).

[26] N. Schnerb, M. Kaveh, Non-Rayleigh statistics of waves in random systems, Phys. Rev. B 43, 1279 (1991).

[27] Th. M. Nieuwenhuizen and M. C. W. van Rossum. Intensity distributions of waves transmitted through a multiple scattering medium, Phys. Rev. Lett., 74, 2674 (1995).

[28] E. Kogan, M. Kaveh, R. Baumgartner, R. Berkovits, Statistics of waves propagating in a random medium. Phys. Rev. B, 48, 9404-9410 (1993).

[29] W. K. Hildebrand, A. Strybulevych, S. E. Skipetrov, B. A. van Tiggelen, J. H. Page, Observation of Infinite-Range Intensity Correlations above, at, and below the Mobility Edges of the 3D Anderson Localization Transition, Phys. Rev. Lett. 112, 073902 (2014).

[30] A. García-Martín, F. Scheffold, M. NietoVesperinas, J. J. Sáenz, Finite-Size Effects on Intensity Correlations in Random Media, Phys. Rev. Lett. 88, 143901 (2002).

[31] I. M. Vellekoop, E. G. van Putten, A. Lagendijk, A. P. Mosk. Demixing light paths inside disordered metamaterials. Opt. Express, 16, 67-80 (2008). 